Internalization of influenza virus and cell surface proteins monitored by site-specific conjugation of protease-sensitive probes

Ross W. Cheloha ${ }^{1}$, Zeyang Li ${ }^{1,2}$, Djenet Bousbaine ${ }^{1,2}$, Andrew W. Woodham ${ }^{1}$, Priscillia Perrin ${ }^{2}$, Jana Volaric ${ }^{1}$, Hidde L. Ploegh ${ }^{1 *}$

${ }^{1}$ Boston Children's Hospital and Harvard Medical School, 1 Blackfan Circle, Boston, MA 02115

${ }^{2}$ Massachusetts Institute of Technology, 455 Main St, Cambridge, MA 02142

* Corresponding author hidde.ploegh@childrens.harvard.edu 


\section{Supporting methods}

General. A20 (ATCC, TIB-208), DC2.4 (Millipore-Sigma, SCC142), and MDCK (ATCC, CCL-34) cell lines were obtained from commercial vendors and cultured under recommended conditions. Cell lines were routinely tested for cell surface marker expression and mycoplasma infection. Detector voltages were not adjusted for analysis. Gating was performed on viable cells based on forward scatter and side scatter profiles.

Peptide synthesis. Peptides were synthesized on Rink-amide linker resin to produce C-terminal amides. Deprotection was performed by exposure to piperidine in dimethylformamide (DMF, 20\% vol/vol) for 15 minutes at room temperature. Coupling was performed using standard Fmoc-protected amino acids (4 equivalents), $\mathrm{N}, \mathrm{N}, \mathrm{N}^{\prime}, \mathrm{N}^{\prime}$-Tetramethyl-O-(1H-benzotriazol-1-yl)uronium hexafluorophosphate (HBTU, 4 equivalents), and diisopropylethylamine (DIPEA, 8 equivalents) in DMF for 45 minutes at room temperature. Conjugation of tetramethylrhodamine to lysine sidechain was performed by on resin sidechain deprotection of Lys $(\mathrm{Mtt})$ by treating with $1.8 \%(\mathrm{v} / \mathrm{v})$ trifluoroacetic acid in dichloromethane. 5(6)-carboxytetramethylrhodamine (TMR, 4 equivalents) and HATU (3.8 equivalents) were dissolved in DMF followed by addition of DIPEA (8 equivalents) and addition to resin with peptide containing sidechain-deprotected lysine for overnight coupling. Following synthesis beads were dried and peptides were cleaved from resin and deprotected in $92.5 \%$ trifluoroacetic acid, $5 \% \mathrm{H}_{2} \mathrm{O}$, and $2.5 \%$ triisopropylsilane (v/v). Peptides were precipitated into cold diethyl ether, pelleted by centrifugation, air-dried, purified using reversed-phase C18 HPLC, and lyophilized. Purified TMR conjugated peptides were then dissolved in DMSO and mixed with equimolar amounts of QSY7-C5-maleimide dissolved in DMSO (ThermoFisher, Q10257) and 10x PBS (1x final concentration PBS). This reaction was monitored by LC/MS, using reversed-phase C18 HPLC, and lyophilized. The identity of peptides was confirmed by LC/MS. Purified products were dissolved in DMSO (10 mM stock concentration) and stored at $-20^{\circ} \mathrm{C}$.

Cell staining and analysis. Suspensions of cells were stained in the presence of $100 \mathrm{nM}$ of the indicated VHH probes in PBS containing $0.5 \%$ bovine serum albumin (BSA) and $0.5 \mathrm{mM}$ EDTA on ice for $1 \mathrm{~h}$. Cells were washed with PBS and resuspended in fetal calf serum (FCS)-supplemented culture medium for the times indicated in the figures. Microscopy was performed on glass bottom microwell dishes (MatTek, No. 1.5 cover glass) or chambered cover glass (LabTek2, 8 well, No. 1.5 cover glass) using either adherent DC2.4 monolayers or A20 cells that were allowed to settle for 30 minutes.

Virus production and labeling. Prior to labeling, $30 \mathrm{~mL}$ of virus stock was concentrated using ultracentrifuge (Beckman SW 55 Ti Swinging-Bucket Rotor) at 36,000 rpm for 2 hours and dissolved in $300 \mu \mathrm{L}$ of rehydration buffer $(0.245 \% \mathrm{BSA}(\mathrm{w} / \mathrm{v}), 150 \mathrm{mM} \mathrm{NaCl}, 20 \mathrm{mM}$ Tris- $\mathrm{HCl}, \mathrm{pH}=7.6)$. Virus was labeled using via sortase-mediated transpeptidation following the protocol described previously with $50 \mu \mathrm{M}$ of peptide probe and $0.5 \mu \mathrm{M}$ sortase. Reaction mixture was incubated at $4^{\circ} \mathrm{C}$ for overnight and purified using sucrose cushion ultracentrifugation. Virus sample was placed on top of sucrose buffer (20\% sucrose, $150 \mathrm{mM} \mathrm{NaCl}, 20 \mathrm{mM}$ Tris- $\mathrm{HCl}, \mathrm{pH}=7.6$ ) and concentrated via ultracentrifugation. Purified virus was dissolved in rehydration buffer. Concentration of the virus was measured using a viral infection assay as previously described ${ }^{1}$. The infectivity of viral preparations before and after sortagging was evaluated through the measurement of cellular levels of influenza nucleoprotein via staining with a nucleoprotein-specific $\mathrm{VHH}$ sortagged with Alexafluor647 as previously described ${ }^{1}$.

Assessment of peptide proteolysis. 
Quenched probes were incubated with recombinant human Cathepsin S (R\&D Systems 1183-CY-010) or human Cathepsin B (Athens 16-12-030102) at $23^{\circ} \mathrm{C}$ according to manufacturer instructions. Briefly, Cathepsin $\mathrm{S}$ was activated through incubation in $\mathrm{pH} 4.5$ sodium acetate $(50 \mathrm{mM})$ buffer with $250 \mathrm{mM}$ $\mathrm{NaCl}$ and $5 \mathrm{mM}$ dithiothreitol (DTT) at a concentration of $10 \mu \mathrm{g} / \mathrm{mL}$ for $2 \mathrm{~h}$ at room temperature and then diluted to the concentration indicated in the figure caption in the same buffer lacking DTT.

Cathepsin B was activated through incubation in pH 5.0 MES (25 mM) buffer with $5 \mathrm{mM}$ dithiothreitol (DTT) at a concentration of $10 \mu \mathrm{g} / \mathrm{mL}$ for $15 \mathrm{~m}$ at room temperature and then diluted to the concentration indicated in the figure caption in the same buffer lacking DTT. Porcine pancreatic trypsin was diluted in $\mathrm{pH}$ 7.4 Tris buffer $(50 \mathrm{mM})$ with $150 \mathrm{mM} \mathrm{NaCl}$ and $10 \%(\mathrm{w} / \mathrm{v})$ glycerol for proteolysis reactions.

Fluorescence spectroscopy of peptides. Fluorescence data was obtained by incubating quenched probes and proteases in a 96-well, black walled plate at $37^{\circ} \mathrm{C}$ for the indicated duration using a Molecular Devices Spectramax M3 plate reader. Samples were excited at $555 \mathrm{~nm}$ and emission was measured at $575 \mathrm{~nm}$.

Michaelis-Menten Kinetics. Kinetic parameters were estimated by incubating concentrations of qP-VVR ranging from $0.6 \mu \mathrm{M}$ to $20 \mu \mathrm{M}$ with fixed concentrations of proteases. Initial velocities were calculated by recording the rate of fluorescence increase observed in the first $2.5 \mathrm{~m}$ after mixing protease and probe. Michaelis-Menten parameters were then calculated using GraphPad Prism 6.

Protein expression. VHHs were expressed using the pHEN6 vector. Plasmids coding for PelB-VHHLPETGG-His 6 were transformed into WK6 E.coli. Bacteria were grown on ampicillin selection at $37^{\circ} \mathrm{C}$ and protein expression was induced with $1 \mathrm{mM} \mathrm{IPTG}$ at $30^{\circ} \mathrm{C}$ overnight. Bacterial pellets were collected and resuspended in TES buffer ( $50 \mathrm{mM}$ Tris, $650 \mu \mathrm{M}$ EDTA, $2 \mathrm{M}$ sucrose, $15 \mathrm{~mL}$ buffer per liter of culture) to prepare for osmotic shock. After incubating for 2 hours at $4{ }^{\circ} \mathrm{C}, 75 \mathrm{ml}$ distilled $\mathrm{H}_{2} \mathrm{O}$ was added and the bacterial suspension was incubated overnight at $4{ }^{\circ} \mathrm{C}$. VHHs were purified from the supernatant by NiNTA bead batch purification, followed by buffer exchange. Sortase-A pentamutant was expressed and purified as previously described ${ }^{2}$.

Protein labeling: VHHs. Sortagging reactions were performed in buffers containing $20 \% \mathrm{DMSO}(\mathrm{v} / \mathrm{v})$ at room temperature for $1 \mathrm{~h}$ with a $1 \mathrm{mM}$ concentration of $\mathrm{G}_{3}$-probe. After the reaction exceeded $50 \%$

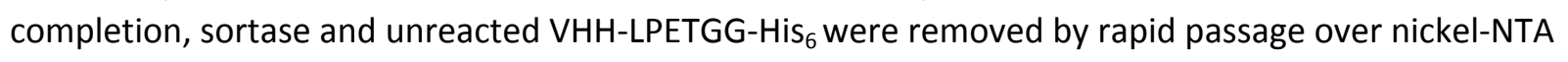
beads. $\mathrm{G}_{3}$-probes were not completely separated from $\mathrm{VHH}$-probe as the products of the sortagging reactions were prone to precipitation upon concentration.

Confocal microscopy to monitor virus internalization. Prior to imaging, MDCK cells at $70 \%$ confluent were split 1:3 and seeded on a live cell imaging chamber with glass bottom (Lab-Tek Chamber Slide, 8 well). After overnight incubation, MDCK cells were infected with virus labeled with different analogs (1250 infectious units per well, 8 well plate) in Opti-MEM serum free media with Hoechst 33342 nucleic acid stain (1:2000) on ice for 1 hour. Unbound virus was removed by washing the wells gently with ice cold Opti-MEM (Zeiss AxioVert 200M) inverted microscope with Yokogawa CSU-22 spinning disk confocal scan head. Images were acquired with MetaMorph software (Molecular Devices) and processed with Volocity (PerkinElmer). 
Lattice light sheet microscopy: The lattice light sheet microscope operates with a manufactured excitation objective (Special Optics, 0.65 NA), a Nikon CFI Api LWD 25x 1.1 NA detection objective, and a Hamamatsu Flash4.0 V2+ camera. The effective pixel size was $0.104 \mu \mathrm{m}$. For generation of the lattice pattern, we used a square lattice and an annular mask with NA $0.42-0.50$. Images were acquired in stage-scanning mode, and deskewed and deconvolved using custom software (cudaDeconv) developed in the Betzig lab at Janelia Research Campus.

One day prior to imaging, MDCK cells were seeded as described for confocal imaging on round $5 \mathrm{~mm}$ coverslips (Warner \#64-0700 CS-5R) followed by incubation with purified virus labeled with different peptide probes and Lyso-tracker (ThermoFisher \#L7526) for $1 \mathrm{~h}$ at $4^{\circ} \mathrm{C}$. Coverslips were then clipped to the end of a long extension sample holder and then dipped into a media bath containing live imaging solution (Thermo Fisher) containing $0.5 \%$ BSA at $37^{\circ} \mathrm{C}$. Images were captured at 17.699 second interval for 45 minutes with excitation at $488 \mathrm{~nm}, 560 \mathrm{~nm}$, and $642 \mathrm{~nm}$. Images were taken as 3D data sets in the $x, y, s$ coordinated system and then were transformed (deskewed) prior to visualization. Images were then processed with Imaris (Bitplane) for time-resolved 3D volume rendering movie.

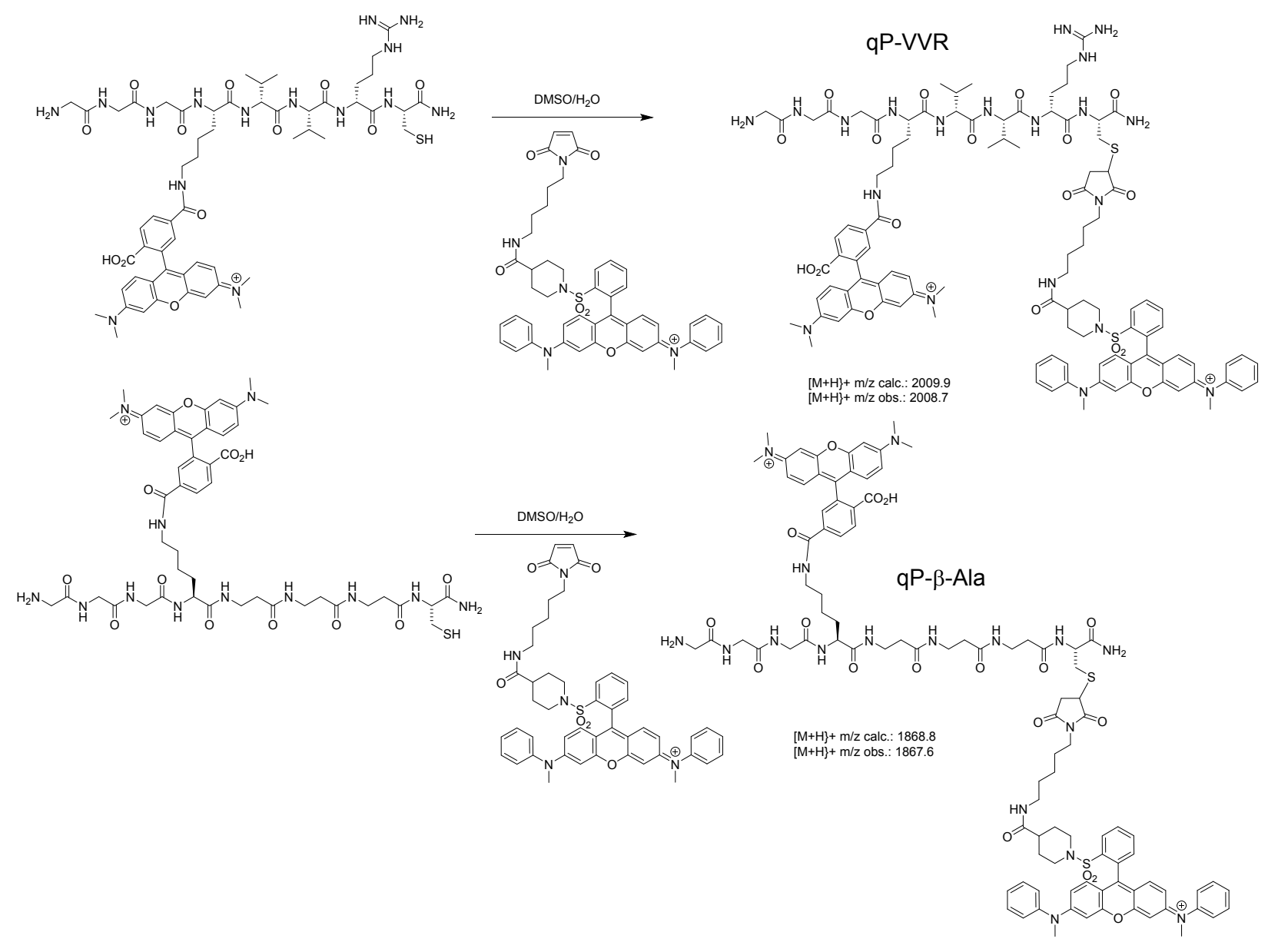



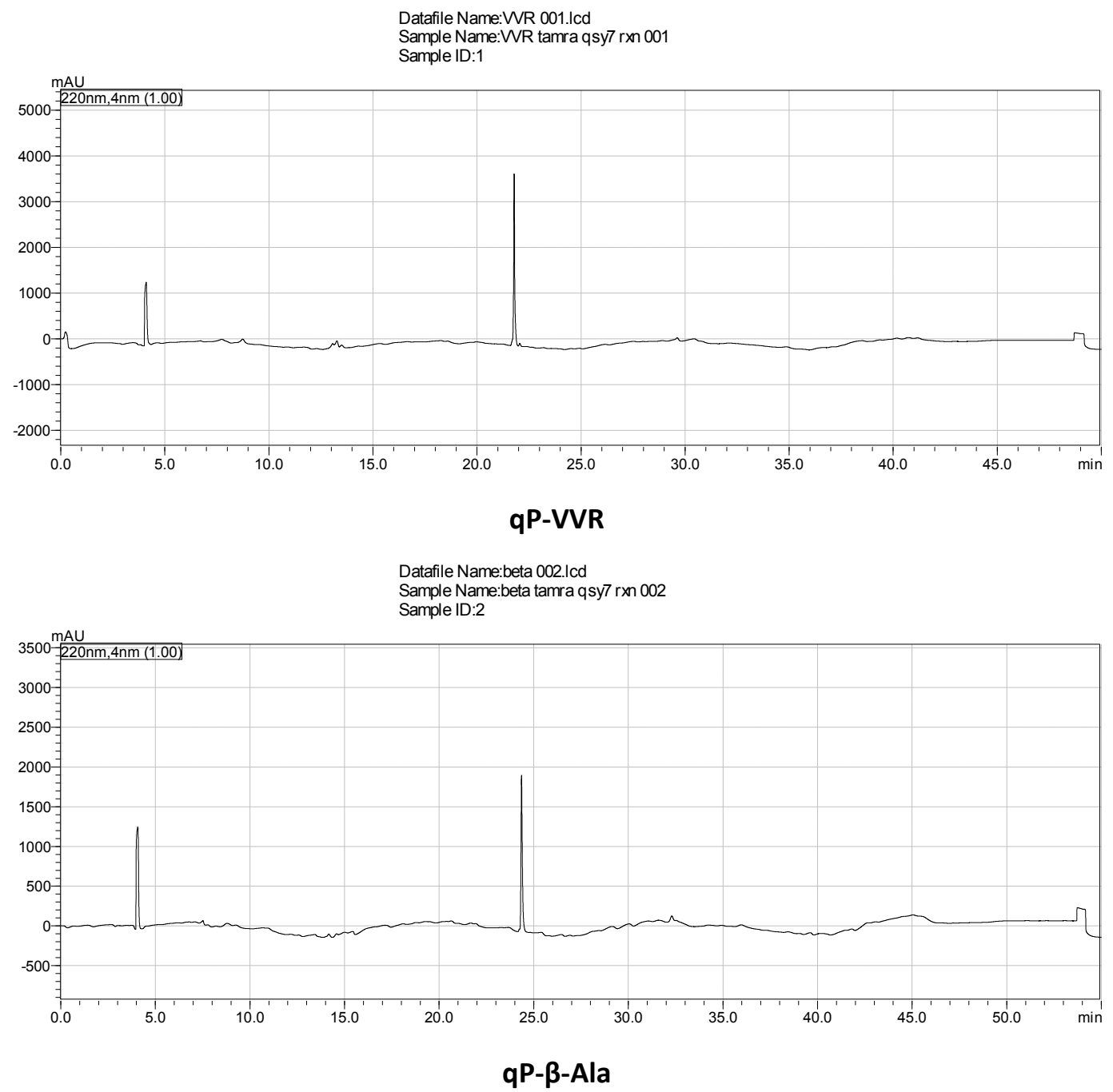

Supporting Figure 1. Scheme for synthesis of quenched probes and analysis. Tetramethyl rhodamine (TMR)-labeled peptides were synthesized using Fmoc-based solid phase peptide synthesis as described in the methods section. Purified TMR-labelled peptides were dissolved in water, mixed with a molar equivalent of maleimide-functionalized fluorescence quencher (QSY7-maleimide) dissolved in DMSO (top). The reaction was monitored by LC/MS and purified by HPLC. The purity of the isolated products was judged to be $>95 \%$ as analyzed by HPLC (bottom). 


\section{CatS CatB Trypsin}

\section{Best-fit values}

$\begin{array}{cccc}\operatorname{Vmax}(\mu \mathrm{mol} / \mathrm{m}) & 619 & 280 & 792 \\ \mathrm{Km}(\mu \mathrm{M}) & 6.1 & 7.1 & 3.7\end{array}$

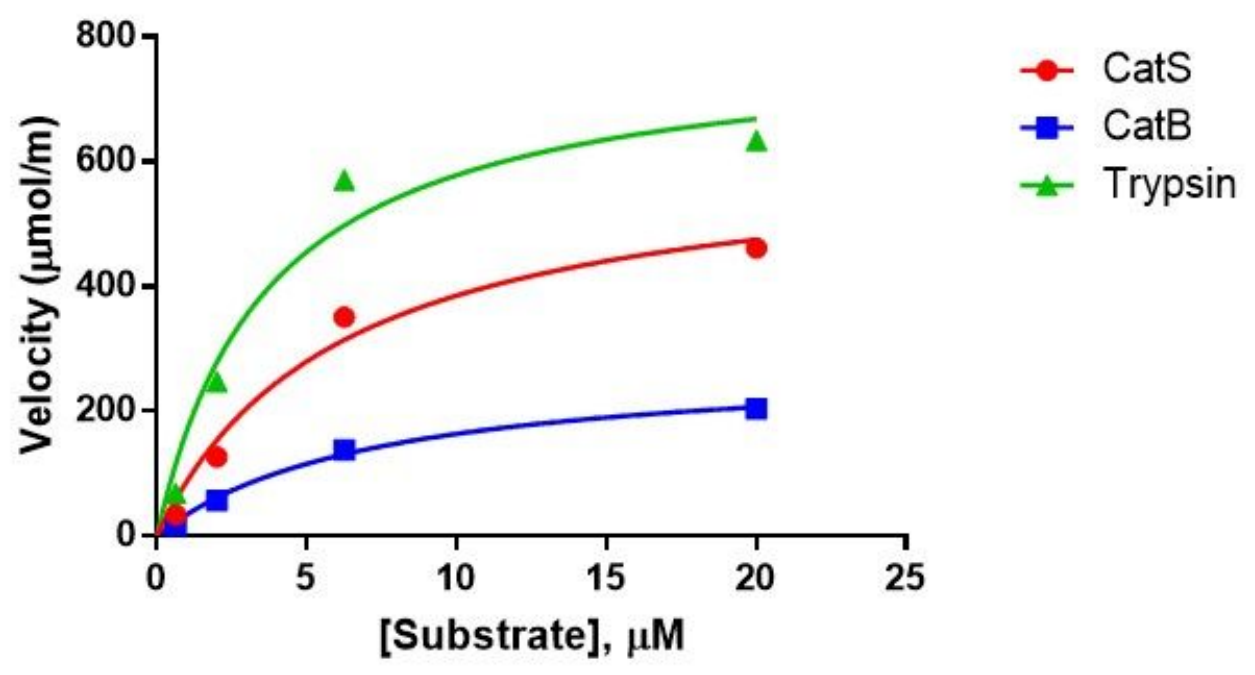

Supporting Figure 2. Michaelis-Menten analysis of cleavage of qP-VVR by selected proteases. Cathepsin $\mathrm{S}(66 \mathrm{nM})$, Cathepsin B (13 nM), or trypsin (43 nM) were incubated with qP-VVR at concentrations ranging from 0.6-20 $\mu \mathrm{M}$. Initial velocities were determined by monitoring the increase in fluorescence at $575 \mathrm{~nm}$ in the first $2.5 \mathrm{~m}$ after mixing probe with protease. Each condition was measured in triplicate. Experimental details are found in the Supporting Methods Section. 
Fluorescence

a)

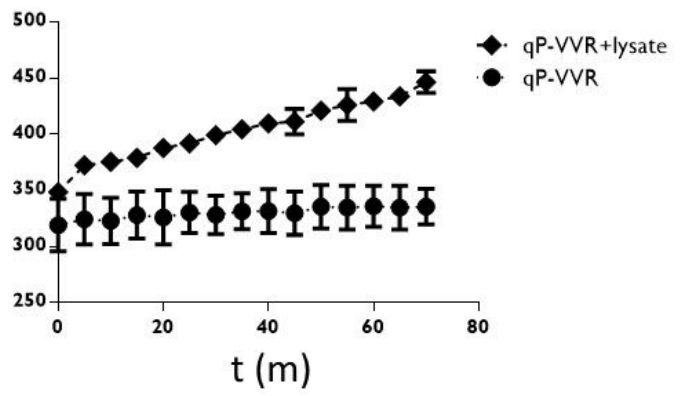

Fluorescence

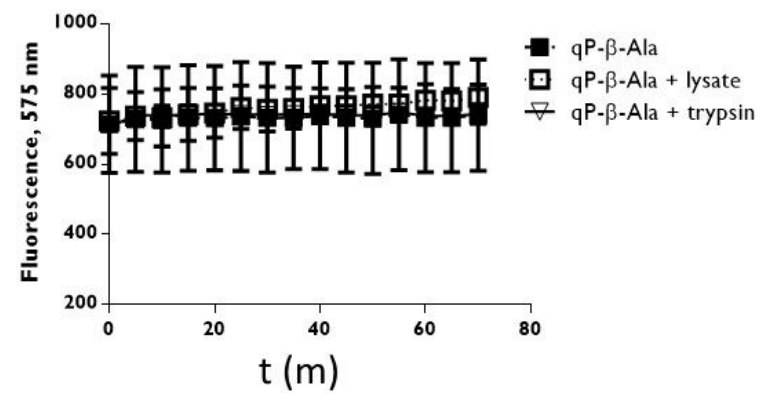

b)

Fluorescence

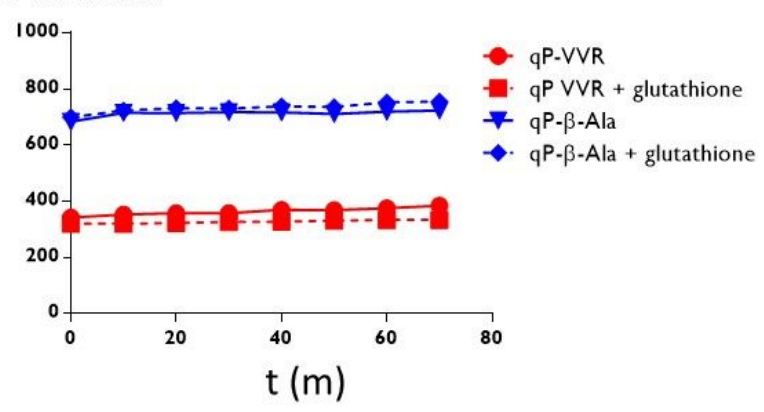

Supporting Figure 3. Dequenching of probes in the presence of (a) A20 cell lysates or (b) reduced glutathione. (a) The indicated probes were dissolved at a concentration of $20 \mu \mathrm{M}$ in either D-PBS or cell lysate (PBS with 0.1\% NP-40). Bovine pancreatic trypsin $(60 \mu \mathrm{M})$ was added to samples dissolved in PBS. Samples in which trypsin was added to solutions of qP-VVR exhibited fluorescence levels of $\sim 7000$ within 1 minute of trypsin addition and are not shown in order to visualize smaller changes in fluorescence. Fluorescence was recorded with excitation at $555 \mathrm{~nm}$ and emission at $575 \mathrm{~nm}$. Error bars represent standard deviation. (b) Experiment performed as in panel a except reduced glutathione (10 $\mathrm{mM})$ was added. 


\begin{tabular}{cc} 
VHH & Observed mass \\
\hline anti-CD47 TMR & 14620 \\
anti-CD47 & 14860 \\
anti-CD47 qP VVR & 15908 \\
anti-CD45 tmr & 14725 \\
anti-CD45 qP VVR & 15985 \\
ani-CD45 & 14950 \\
anti-Kappa & 15160 \\
anti-Kappa qP VVR & 16235 \\
anti-Kappa tmr & 14955 \\
anti-MHC-II & 15620 \\
anti-MHC-II qP bAla & 16530 \\
anti-MHC-II qP VVR & 16690 \\
anti-MHC-II tmr & 15395 \\
anti-CD36 qP VVR & 16305 \\
anti-CD36 & 15255 \\
anti-CD36 tmr & 15025 \\
anti-GFP tmr & 14005 \\
anti-GFP qP VVR & 15285 \\
anti-GFP & 14235
\end{tabular}

Supporting Figure 4. Characterization of VHH-probe conjugates with mass spectrometry. Molecular weights were quantified on a Waters LC/qTof-MS using MaxEnt deconvolution. 
a)

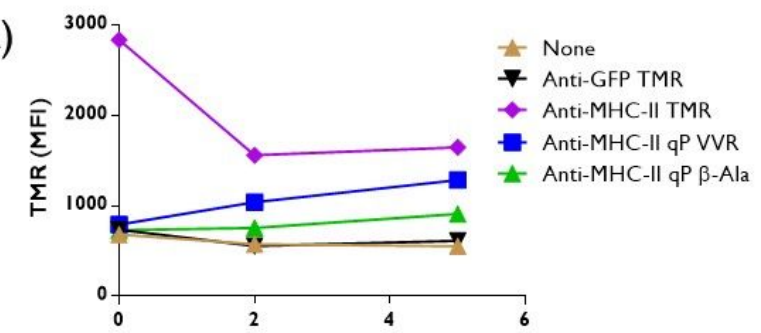

c)

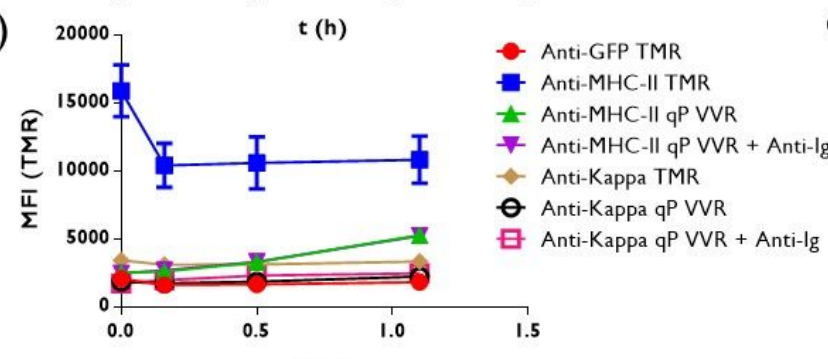

e)

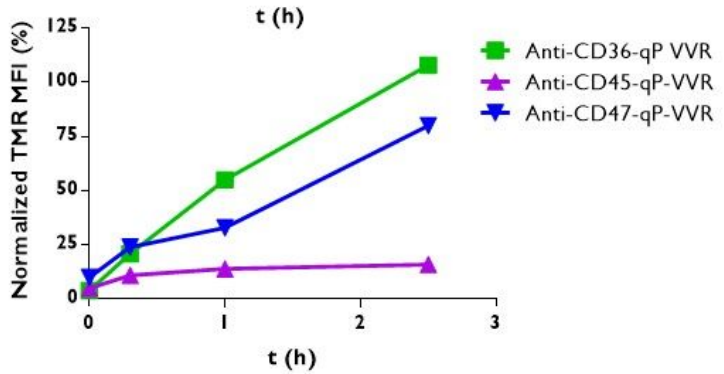

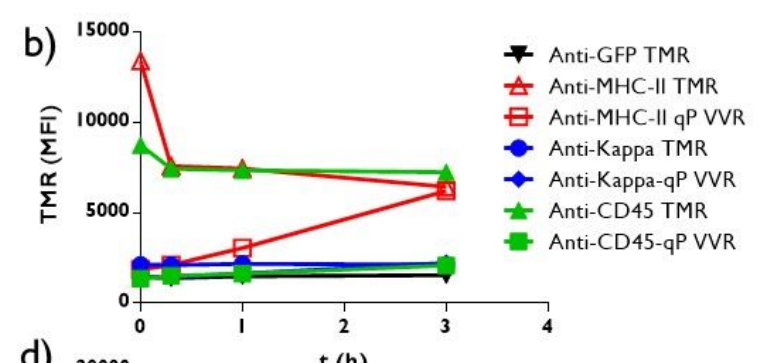

d) $20000 \quad t(h)$

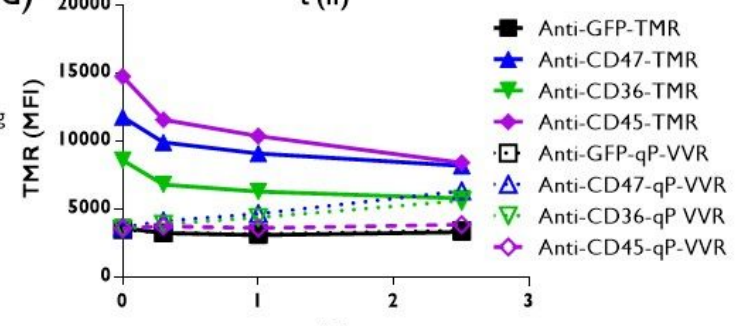

f)

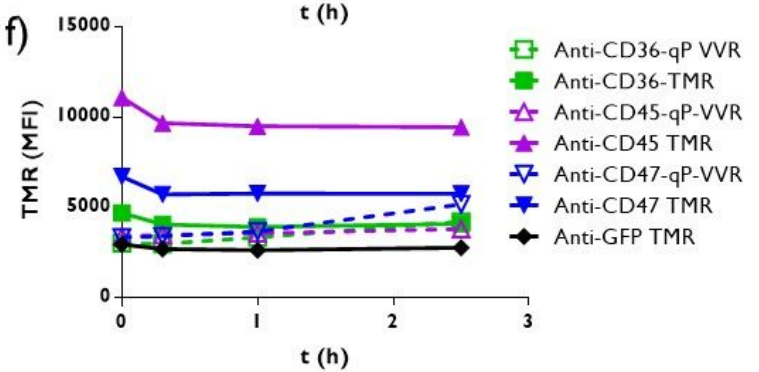

Supporting Figure 5. Raw data and experimental replicates for data from Figure 3. (a-d) Raw data for main Figures 3a-d. (e-f) Replicate experiment for internalization of probes in DC2.4 cells. MFI values are plotted as normalized units (e) or as raw median fluorescence intensities ( $f$ ). 


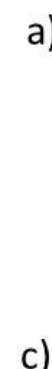

a)

c)
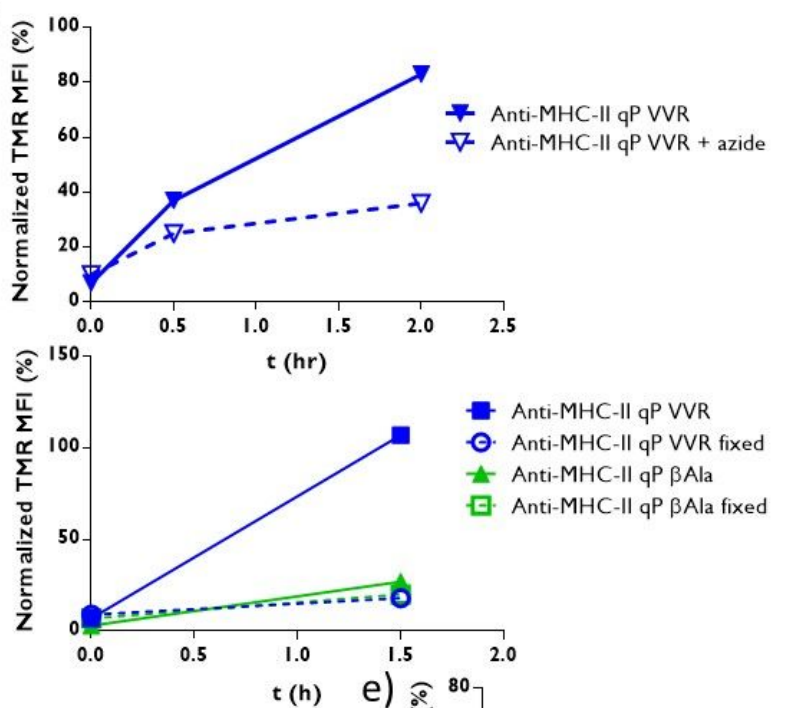

b) 5000

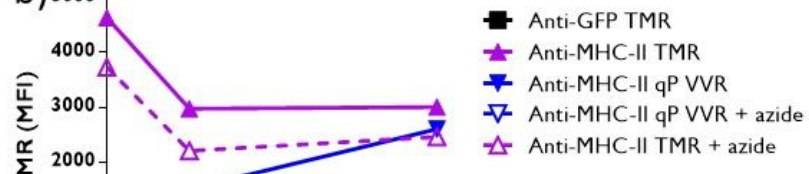

d)

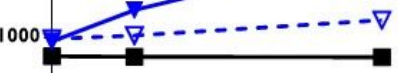

$\triangle$ Anti-MHC-II TMR + azide
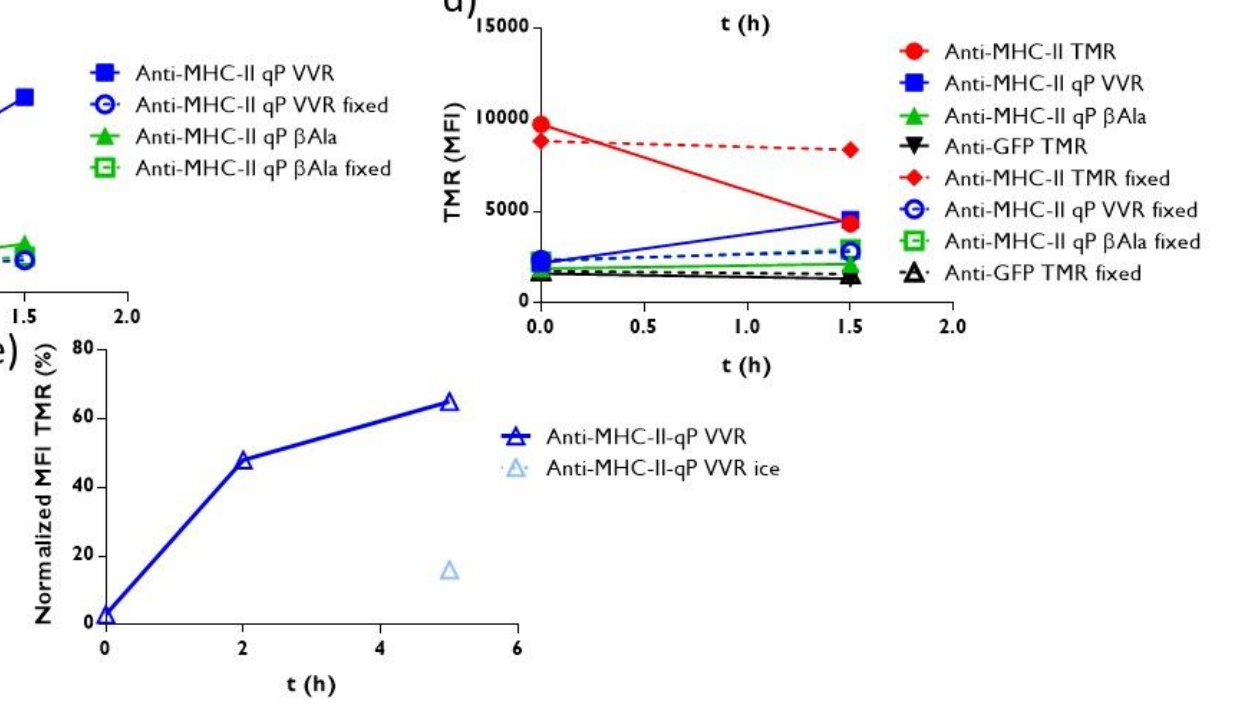

Supporting Figure 6. Treatment of A20 cells with azide or fixation with paraformaldehyde slows dequenching and internalization. (a-b) A20 cells were stained with the indicated conjugates and treated with sodium azide ( $120 \mathrm{mM}$ ) for $1 \mathrm{~h}$ on ice. Cells were centrifuged, washed and resuspended in culture media containing azide at $37^{\circ} \mathrm{C}$ for the indicated durations. Data from panel b were normalized to provide data in panel a as described in the methods section. (c-d) A20 cells were stained with the indicated conjugates on ice, centrifuged, washed, then fixed using $4 \%$ paraformaldehyde. Cells were resuspended in culture media at $37^{\circ} \mathrm{C}$ for the indicated durations. Data from panel $\mathrm{d}$ were normalized to provide data in panel $\mathrm{c}$ as described in the methods section. (e) A20 cells were stained with the indicated conjugates, centrifuged, and resuspended. Cells were either incubated at $37^{\circ} \mathrm{C}$ or kept on ice for the indicated durations 
a)

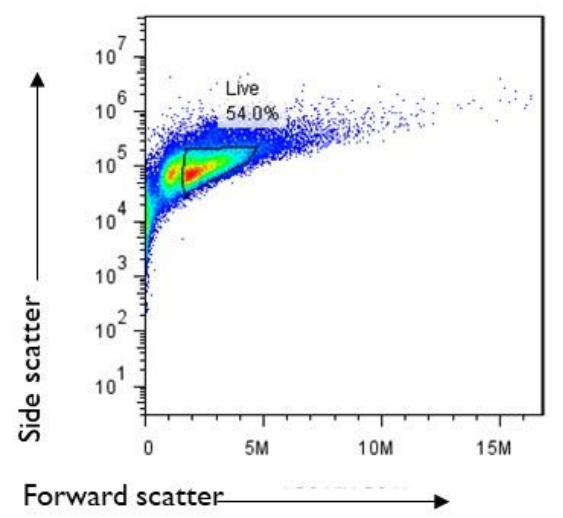

c)

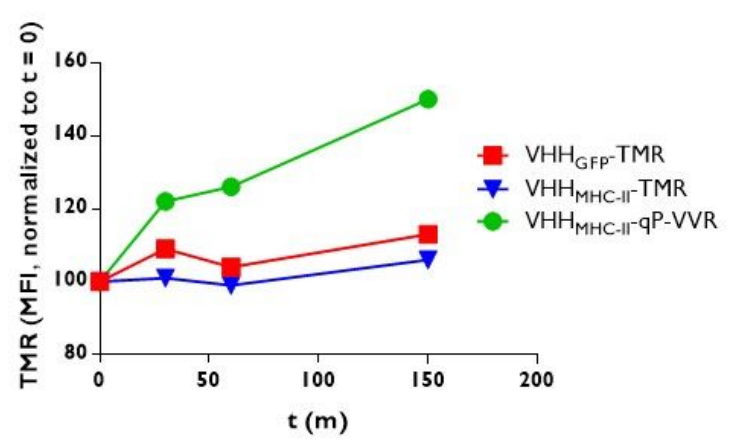

b)

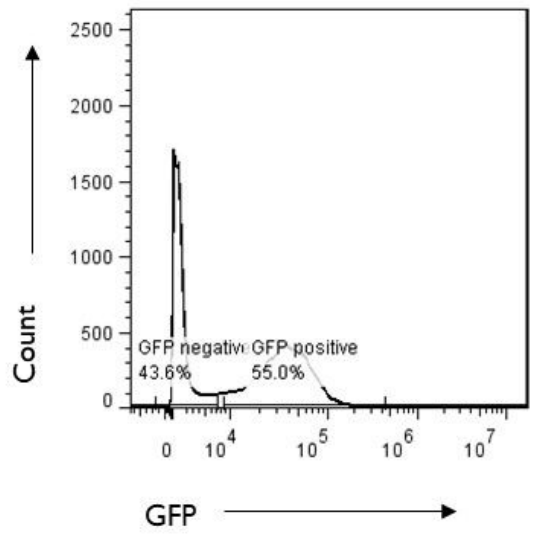

d)

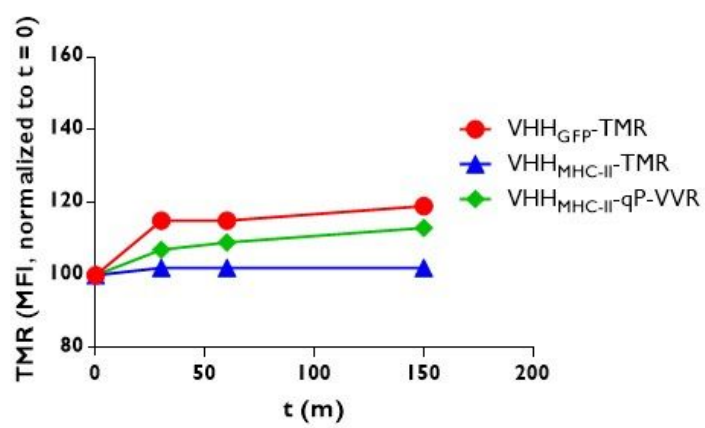

Supporting Figure 7. Anti-MHC-II $\left(\mathrm{VHH}_{\mathrm{MHC}-I I}\right)$ conjugated with quenched probe retains specificity for MHC-II positive splenocytes. Freshly prepared murine splenocytes from MHC-II GFP mice were stained in PBS containing $0.5 \%$ BSA and $0.5 \mathrm{mM}$ EDTA for $1 \mathrm{~h}$ on ice, washed, and resuspended in RPMI complete for the indicated durations by flow cytometry. (a) Gating to select live cells for analysis. (b) Gating to stratify live MHC-II-GFP positive cells from MHC-II-GFP negative cells. (c-d) Plots of changes in TMR median fluorescence intensities over time in (c) GFP positive or (d) GFP negative cell populations. TMR MFIs are normalized to values obtained immediately after staining on ice $(t=0)$ to enable comparison between different cell populations. 


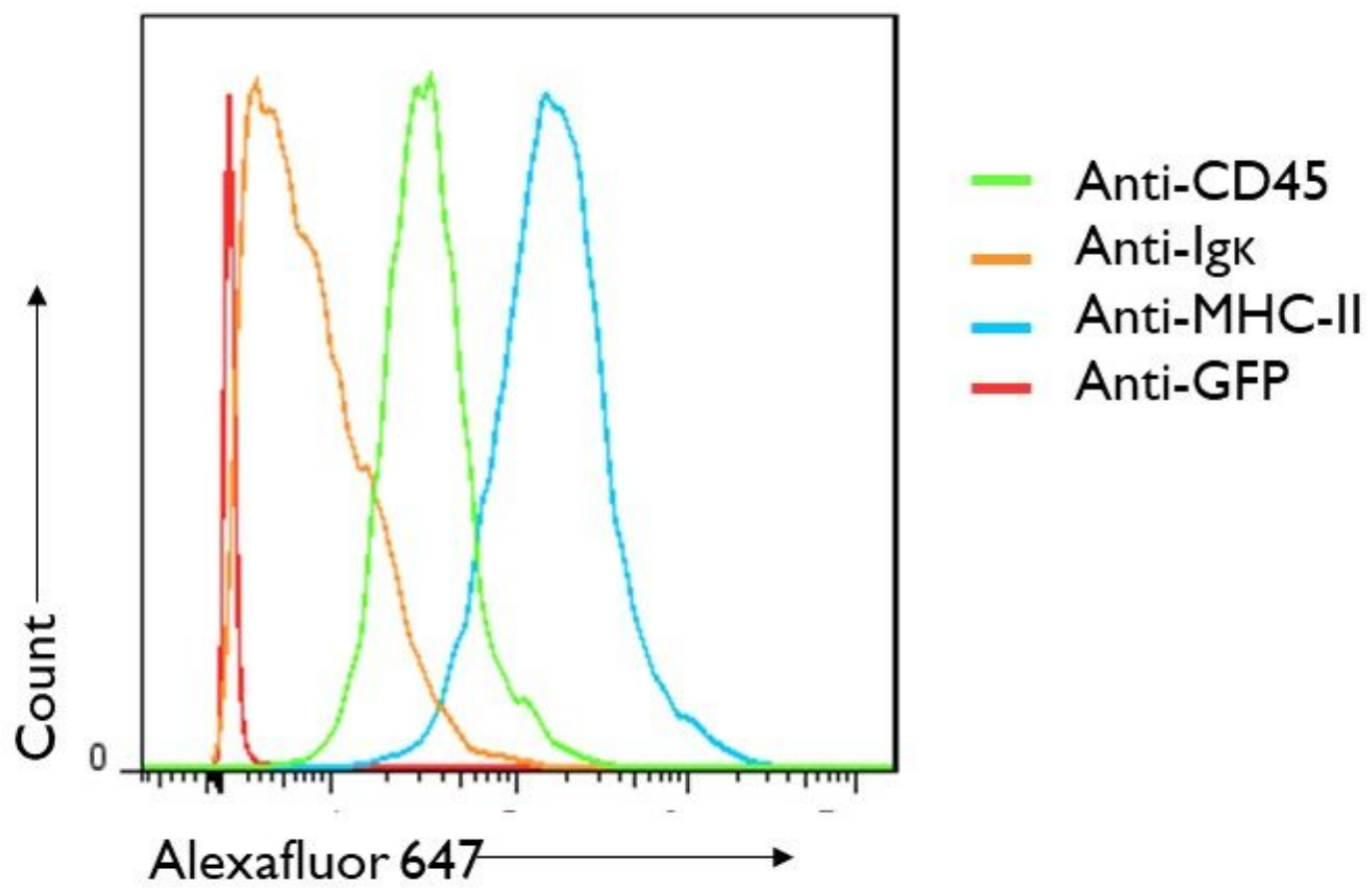

Supporting Figure 8. A20 cells are stained by VHHs that recognize CD45, Igk, and MHC-II sortagged with Alexafluor 647. A20 cells were stained in PBS containing 1\% BSA and 0.5 mM EDTA for $1 \mathrm{~h}$ on ice, washed, and analyzed by flow cytometry. Cells were gated on $\mathrm{FSC} / \mathrm{SSC}$ to exclude ruptured cells. 
a)

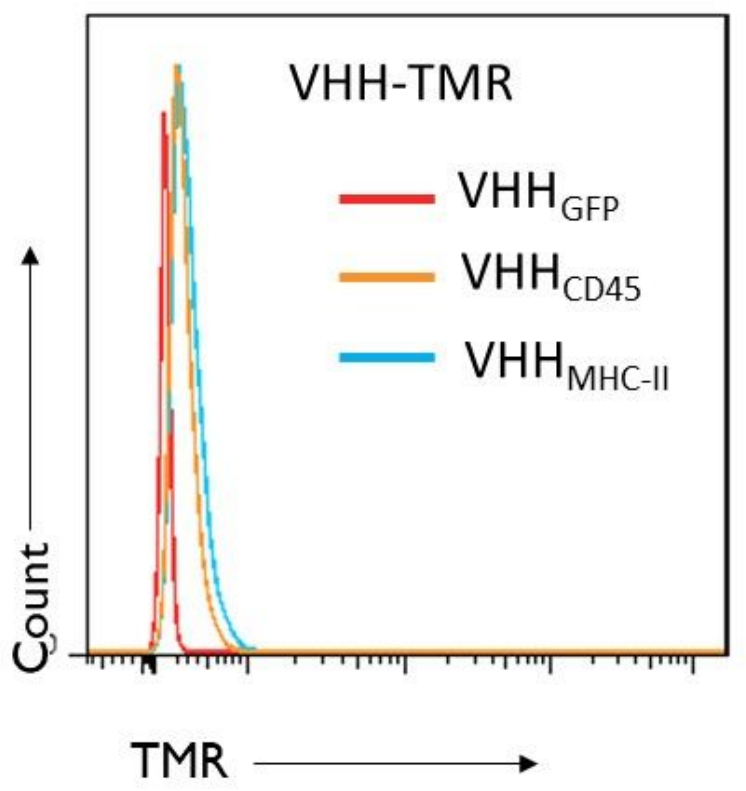

c)

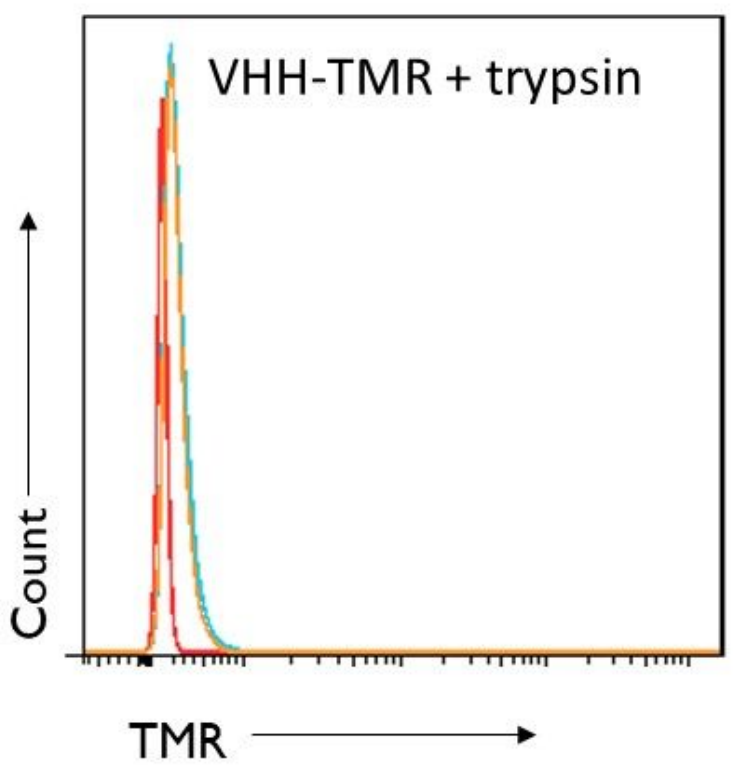

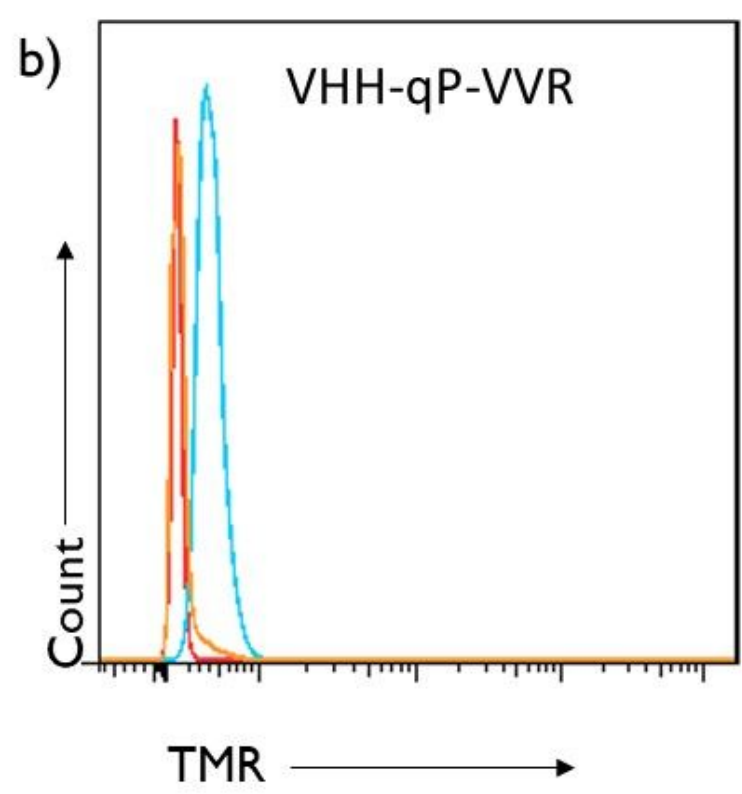

d)

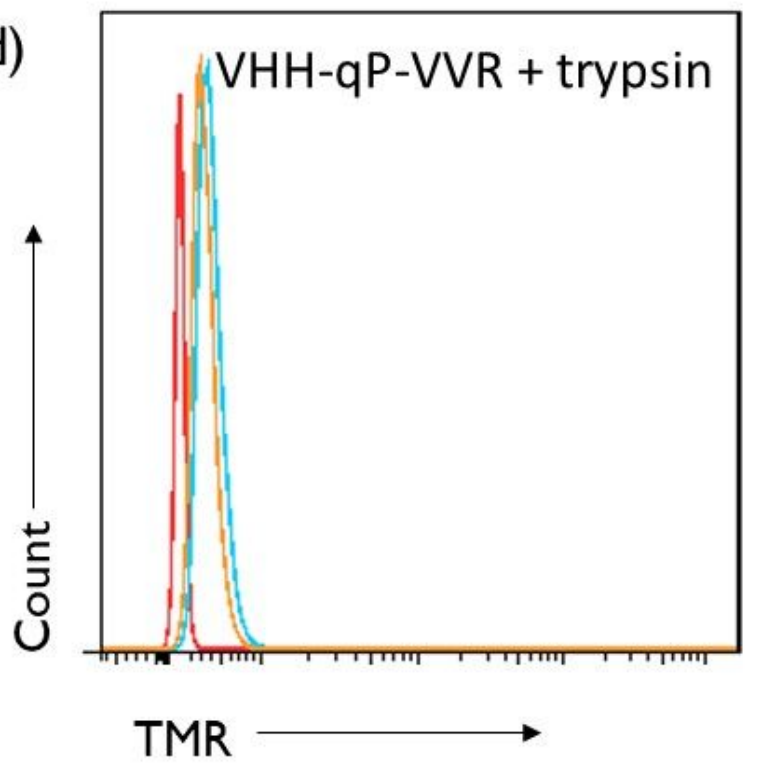

Supporting figure 9. Cell surface associated $\mathrm{VHH}_{\mathrm{CD} 45}-\mathrm{qP}-\mathrm{VVR}$ remains cell bound throughout the experiment and is not cleaved by secreted proteases. A20 cells were labeled with VHHs sortagged with TMR or qP-VVR were used to label A20 cells as described in the methods section. These labeled cells were incubated in culture medium for $3 \mathrm{~h}$ at $37^{\circ} \mathrm{C}$ and then analyzed by flow cytometry (panels a, b) or centrifuged, washed, treated with $0.004 \%$ trypsin and then analyzed by flow cytometry (panels $c, d$ ). The $\mathrm{VHH}$ used for staining is indicated by the color of the trace in the histogram and is identical for all panels. Samples were gated on intact cells by forward scatter and side scatter. 

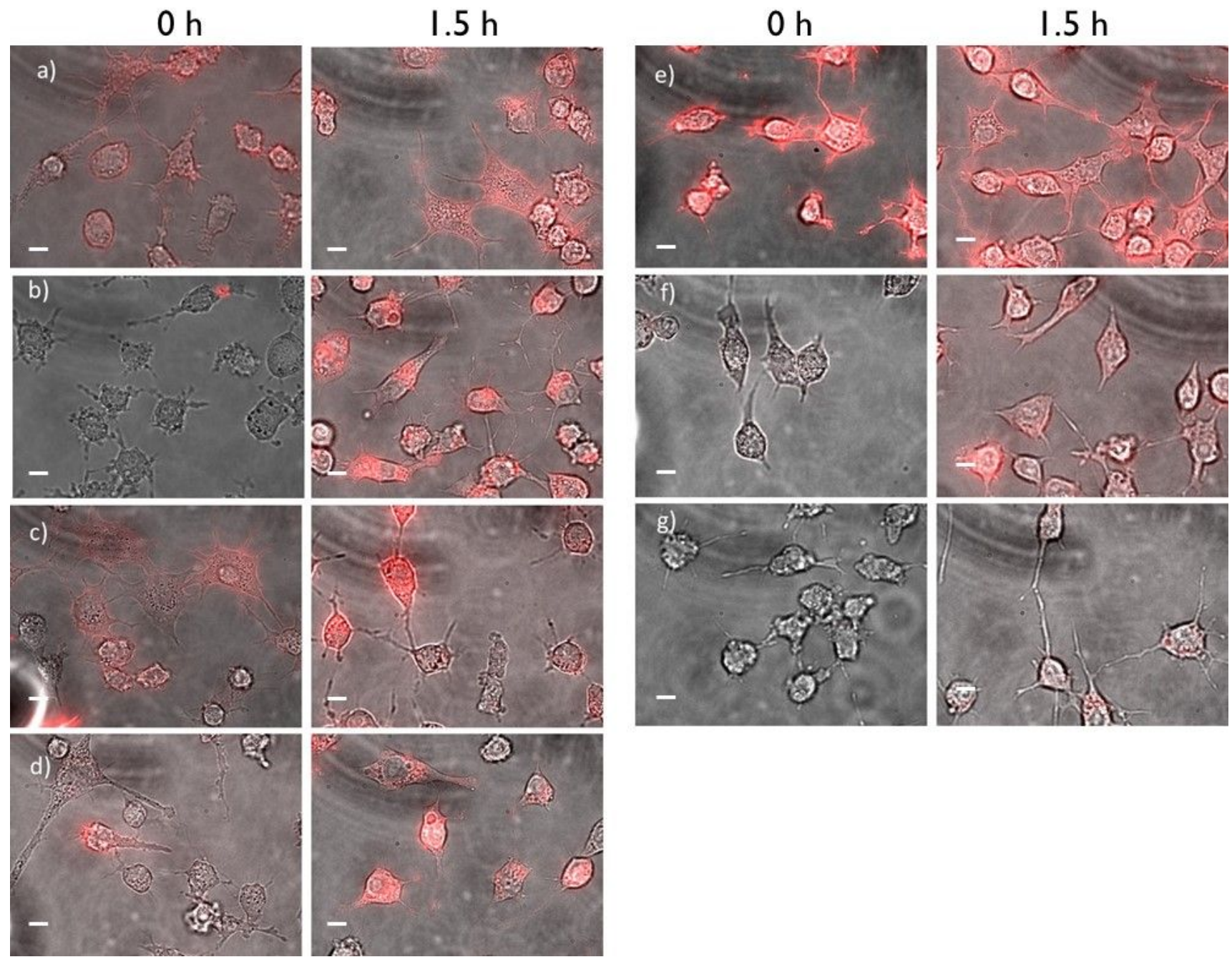

Supporting Figure 10: Analysis of quenched fluorophore probes using fluorescence microscopy. DC2.4 cells were stained with fluorophore or probe-conjugated VHH for 1 hour on ice and imaged immediately (left) or following incubation at $37^{\circ} \mathrm{C}$ for $1.5 \mathrm{~h}$ (right). The VHHs used for staining are (a) Anti-CD47-TMR (b) Anti-CD47-qP VVR (c) Anti-CD36-TMR (d) Anti-CD36-qP VVR (e) Anti-CD45-TMR (f) Anti-CD45-qP VVR and (g) Anti-GFP-TMR. Scale bars represent $10 \mu \mathrm{m}$. 


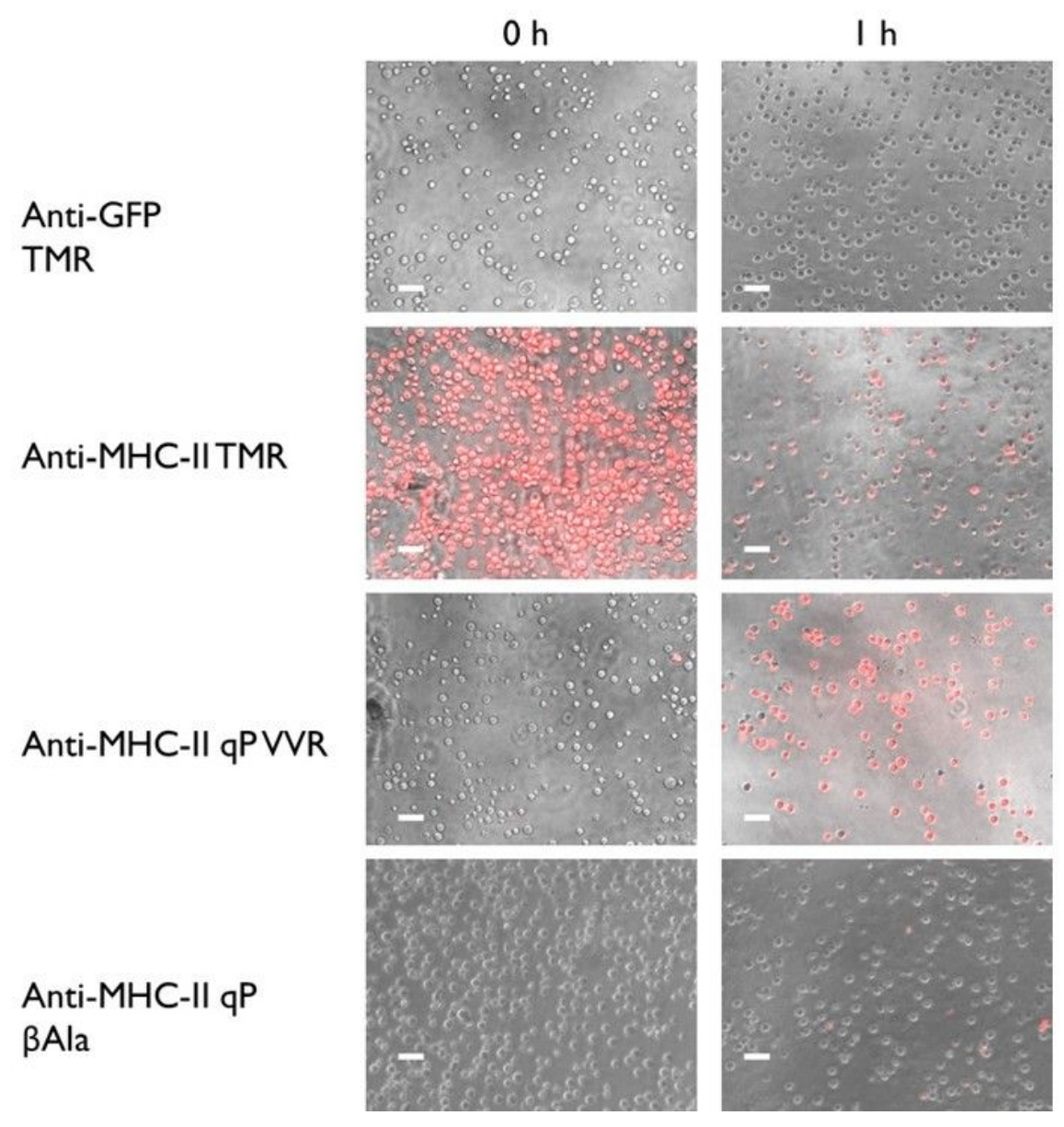

Supporting Figure 11: Analysis of quenched fluorophore probes using fluorescence microscopy. A20 cells were stained with fluorophore or probe-conjugated $\mathrm{VHH}$ for 1 hour on ice and imaged immediately (left) or following incubation at $37^{\circ} \mathrm{C}$ for $1 \mathrm{~h}$ (right). The $\mathrm{VHHs}$ used for staining are indicated to the left of the pair of images. Scale bars represent $50 \mu \mathrm{m}$. 


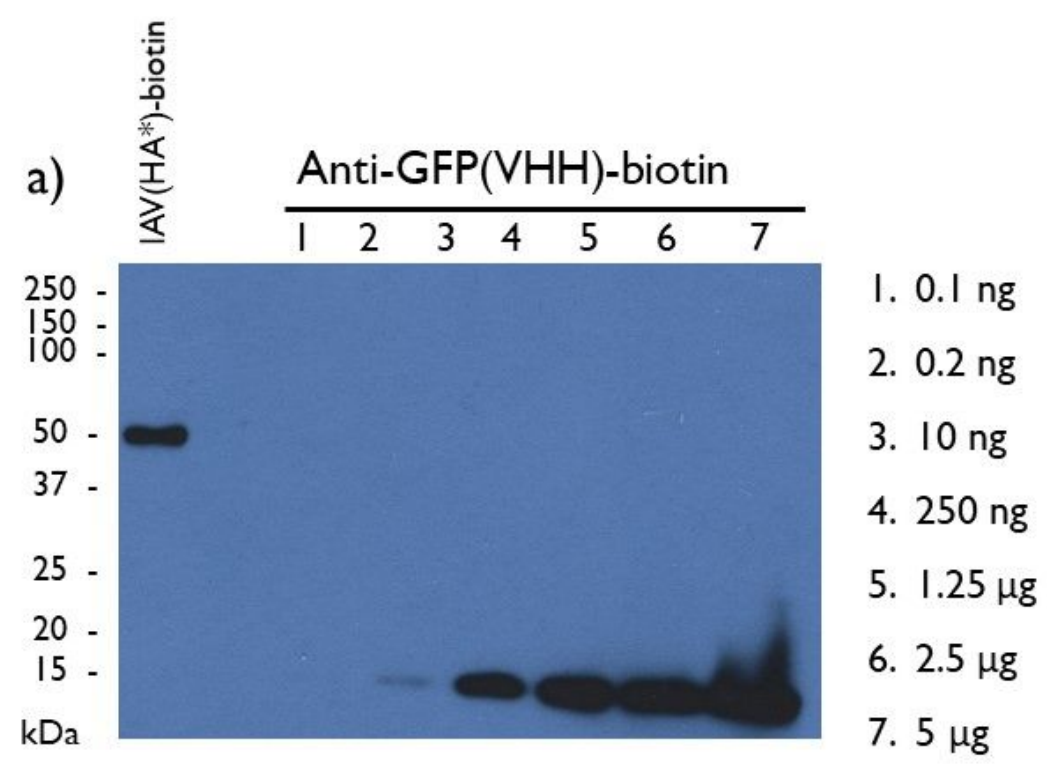

b)

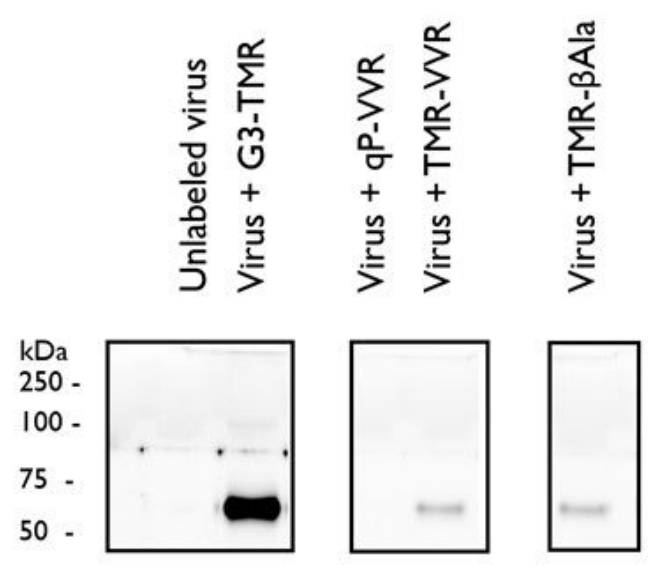

Fluorescence $(555 / 575 \mathrm{~nm})$

Supporting Figure 12. Labeling of intact influenza A viral particles with probes using sortase. (a) Influenza A expressing HA-LPETG [IAV(HA*)] was incubated with $\mathrm{G}_{3}$-biotin $(50 \mu \mathrm{M})$ and sortase $\mathrm{A}$ pentamutant $(5 \mu \mathrm{M})$ in the presence of $10 \%$ DMSO overnight at $4^{\circ} \mathrm{C}$. Labelled virus was subjected to SDS-PAGE, transfer to a PDVF membrane and visualized using streptavidin-HRP conjugate. $\mathrm{VHH}_{\mathrm{GFP}}$ was uniformly labeled with a single biotin using sortagging as described in the methods section and was used for loading controls. (b) IAV(HA*) was labeled with either $\mathrm{G}_{3}-T M R$, qP VVR, or TMR VVR as described in panel $A$. Labeled proteins were resolved by SDS-PAGE and visualized by fluorescence gel scanning. 


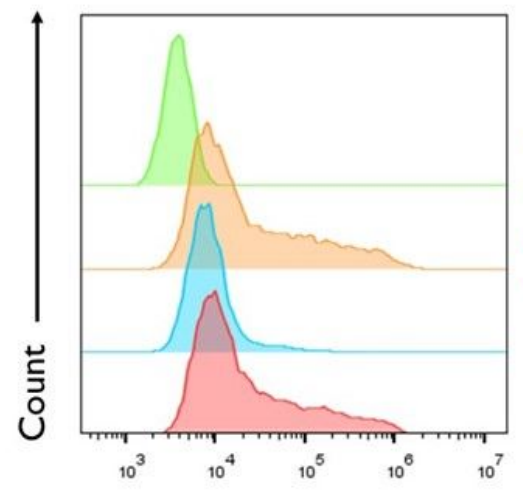

- Unstained control

- qP-BAla virus

- Irrelevant peptide-virus

- qP-VVR virus
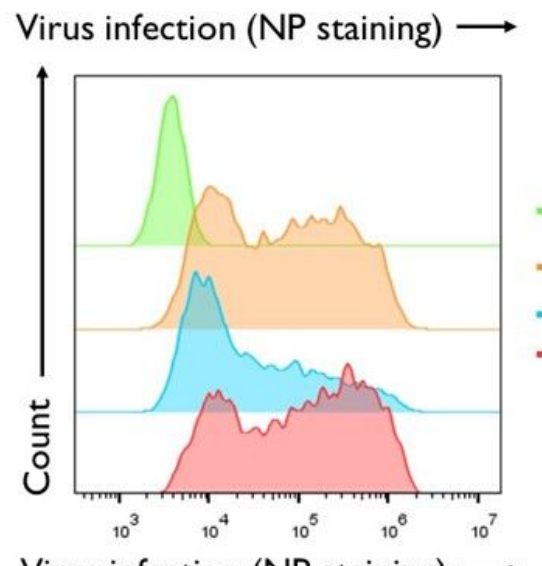

- Unstained control

- TMR-BAla virus

- Irrelevant peptide-virus

- TMR-VVR virus

Virus infection (NP staining) $\longrightarrow$

Supporting Figure 13. Influenza A expressing HA-LPETG remains infectious following labeling with quenched probes. Labeled virus was produced as described in the supporting methods section. MDCK cells were exposed to virus at a multiplicity of infection of approximately 10 . Six hours after virus addition infection was analyzed by flow cytometry.

[Video file separate]

Supporting Figure 14. Visualization of quenched-probe functionalized virus internalization using lattice light sheet microscopy. Influenza A expressing HA-LPETG was labeled with qP-VVR and applied to MDCK cells as described in the methods section. Images were acquired and processed as described in the methods section. Nuclear staining (Hoechst) is blue, lysosomal staining (lyso-tracker) is green, and virus with probe that has been dequenched upon internalization is red (qP-VVR). The total duration of image acquisition was 43 minutes. Each second of the movie corresponds to 5.3 minutes in real time. 